\title{
CARACTERIZACIÓN DE LOS SISTEMAS PRODUCTIVOS DE CAFÉ EN NARIÑO, COLOMBIA
}

\section{CHARACTERIZATION OF COFFEE PRODUCTION SYSTEMS IN NARIÑO, COLOMBIA}

\author{
Hernando Criollo Escobar ${ }^{1}$, Tulio César Lagos Burbano ${ }^{2}$, Tito Bacca Ibarra ${ }^{3}$, Johanna Alixa Muñoz Belalcazar ${ }^{4}$
}

\begin{abstract}
${ }^{1}$ Ing. Agrónomo, Ph.D., profesor asociado, fisiología vegetal. Universidad de Nariño, Facultad de Ciencias Agrícolas, Grupo

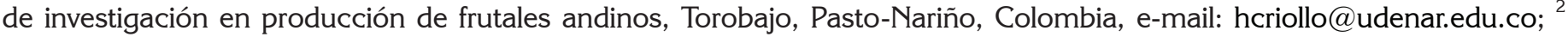
Ing. Agrónomo, Ph.D., profesor titular, mejoramiento genético. Universidad de Nariño, Facultad de Ciencias Agrícolas, Grupo de investigación en producción de frutales andinos, Torobajo, Pasto-Nariño, Colombia, e-mail: tclagosb@udenar.edu.co; ${ }^{3}$ Ing. Agrónomo, Ph.D., profesor titular, entomología. Universidad del Tolima, Sede Santa Helena Parte Alta, Ibagué-Tolima, Colombia, e-mail: titobacca@ut.edu.co; ${ }^{4}$ Ing. Agrónomo, M.Sc. Grupo de investigación en producción de frutales andinos, Torobajo, Pasto-Nariño, Colombia, e-mail: mjohannaalixa@yahoo.es
\end{abstract}

Rev. U.D.C.A Act. \& Div. Cient. 19(1): 105-113, Enero-Junio, 2016

\section{RESUMEN}

El objetivo fue caracterizar los sistemas productivos de café en los ecotopos 220A y 221A, de acuerdo con la clasificación establecida por la Federación Nacional de Cafeteros localizados en el departamento de Nariño. En cada uno de estos ecotopos, se estudiaron los sistemas productivos de café, localizados a menos de $1500 \mathrm{msnm}$, entre 1501 y $1800 \mathrm{msnm}$ y por encima de $1800 \mathrm{msnm}$. Se utilizaron variables categorizadas relacionadas con el sistema productivo de café, tomadas de 159 encuestas a productores, identificados aleatoriamente, desde un marco muestral de 16.767 unidades productivas cafeteras. Estas variables, se sometieron a los análisis multivariados de correspondencias múltiples y análisis de clasificación jerárquica. El ecotopo 220A, se caracterizó por poseer buenas vías de acceso, cultivos de café con edades menores a seis años, con áreas de una a tres hectáreas. Los cultivos de café de este ecotopo están asociados con árboles de naranja y guamos. El Ecotopo 221A tiene buenas vías de acceso, los cafetales están asociadas con frutales, principalmente, con plátano y con banano; igualmente, se observó que el 9,2\% de los cultivos se plantan sin sombrío. La fertilización y el control de plagas son eminentemente químicos. En ambos ecotopos, la mayoría de los productores fertilizan sin realizar previamente análisis de suelo, predominan los cultivos asociados sin un ordenamiento aparente, que permita manejar adecuadamente los niveles de sombrío.

Palabras clave: Coffea arabica L., sombra permanente, análisis multivariado, ecotopo.

\section{SUMMARY}

The aim of this research was to characterize the coffee production systems in ecotopes 220A and 221A, classification established by the National Coffee Growers Federation, in the department of Nariño. In each of these ecotopes the coffee production systems located at less than 1500 meters above sea level, between 1501 and $1800 \mathrm{~m}$ and above $1800 \mathrm{~m}$ were studied. Variables related to the productions system were evaluated through surveys of 159 growers, taken randomly from a sampling frame of 16767 coffee production units. These variables were subjected to multiple correspondence analysis and hierarchical classification. The ecotope $220 \mathrm{~A}$ is characterized by having good access roads, coffee plantations younger than six years, comprising areas from one to three hectares. The coffee of this ecotope is associated with orange and guamos trees. The ecotope 221A, has good access roads, the coffee plantations are associated with fruit trees, mainly bananas and cooking bananas. Results showed that $9.2 \%$ of the crops are grown without shade. Fertilization and pest control are principally made by chemical means. In both ecotopos, most producers fertilize without knowing soil analyses results; in the area predominate coffee crops grown in associations, in no apparent order, which would manage shade levels.

Key words: Coffea arabica L., permanent shade, multivariate analysis, ecotope. 


\section{INTRODUCCIÓN}

Para el 2013, Colombia produjo 10.900 .000 sacos de café de $60 \mathrm{~kg}$, que representan el $7,4 \%$ de la producción mundial, siendo el cuarto país productor de café en el mundo, después de Brasil, Vietnam e Indonesia (OIC, 2014). Urueña (2013) indica que el cultivo de café tiene gran importancia en la economía colombiana, constituyendo el 15\% del PIB agropecuario, para el 2012; además, se caracteriza por poseer cafetales de ocho años de edad, con densidades promedio de 5.015 plantas por hectárea.

Para el 2013, Colombia registra 771.731 ha cultivadas en café, de las cuales, el departamento de Nariño aporta 32.137 ha, que representan el 4,16\% (Agronet, 2015), cuya producción es muy apreciada en el comercio internacional, por su denominación de origen (Oberthür et al. 2011). En Nariño, el 87,3\% de la caficultura es tecnificada, con árboles jóvenes de 2 a 8 años, variedades resistentes a la roya en un $52,6 \%$ (Saldías, 2013) y un promedio de rendimiento de 0,9t. $\mathrm{ha}^{-1}$, superior al promedio nacional $\left(0,8 \mathrm{t} \cdot \mathrm{ha}^{-1}\right)$.

En el país, los cafetales se desarrollan en un rango altitudinal de 1000 a $2000 \mathrm{msnm}$, la temperatura promedio es de $20^{\circ} \mathrm{C}$ y la precipitación puede oscilar entre 1440 a $2711 \mathrm{~mm} /$ año. Estas condiciones han variado en los últimos años, debido a la variabilidad del clima, a los eventos del niño y la niña, según lo expresan Ramírez et al. (2013). Estos fenómenos, junto con los factores genéticos de la planta y los sistemas de manejo del cultivo, determinan la productividad del café. De esta actividad cafetera, dependen económicamente 563.000 productores, que se caracterizan, principalmente, por la producción familiar de minifundio (Saldías, 2013).

Según Gómez et al. (1991), el Centro Nacional de Investigaciones del café (CENICAFE), con base en las clasificaciones de suelos del país del Instituto Geográfico Agustín Codazzi IGAC, del Instituto Colombiano de Hidrología, Meteorología y Adecuación de Tierras (HIMAT), del Instituto Colombiano Agropecuario ICA, que utilizaron en su metodología variables agrícolas, balances hídricos decadales, áreas agroecológicas y fisiografía, subdividió la zona cafetera colombiana en 86 ecotopos cafeteros, caracterizados por el sistema geográfico, variables climáticas, variables del suelo y cartografía (Torrente \& Ladino, 2009).

En el departamento de Nariño, de 64 municipios que lo conforman, 41 son productores de café. La zona norte representa el $66,63 \%$, mientras que en el suroccidente, se encuentra el 33,37\% del total de predios dedicados a este cultivo. Gómez et al. (1991) identificaron en el departamento dos zonas agroecológicas, con características agroclimáticas similares, que corresponden a los ecotopos 220A y 221A.
El ecotopo 220A, ubicado en el norte del departamento, comprende las regiones ubicadas en la cuenca del río Patía, en las subcuencas de los ríos Juanambú y Mayo, en las coordenadas que van desde $1^{\circ} 21^{\prime}$ hasta $1^{\circ} 42$ 'LN, altitudes de 1.300 a $1.800 \mathrm{msnm}$ y una precipitación anual de 1.700 a $1.900 \mathrm{~mm}$. El ecotopo 221A, se encuentra localizado en las zonas que hacen parte de la cuenca del río Guáitara, entre $1^{\circ} 05^{\prime}$ y $1^{\circ} 36$ 'LN, entre 1.400 y $2.100 \mathrm{msnm}$ y con una precipitación anual de 1.400 a $1.700 \mathrm{~mm}$ (FNC,1991).

Existen grandes diferencias en las zonas cafeteras de los ecotopos indicados, que se relacionan con el área sembrada, sistemas de siembra, programas de fertilización y, en general, en todas las actividades relacionadas con la producción del cultivo y la poscosecha. Por esta razón, es importante caracterizar física y socialmente, las fincas cafeteras de estos ecotopos, para formar grupos, establecer las diferencias entre ellos y definir sus principales características.

La caracterización y el análisis de las zonas productoras, constituyen métodos importantes para determinar su distribución y cuantificar las características e interacciones que determinan su funcionamiento; además, permiten cuantificar las diferencias, mediante la descripción de las propiedades de un sistema y de sus interacciones, facilitando así la planeación y la optimización de los recursos, orientados al mejoramiento de las unidades productivas (Ríos et al. 2004; Merma \& Julca, 2012).

La caracterización de los sistemas productivos de café reconoce variables que contribuyen al desarrollo sostenible de este cultivo, por ejemplo, Rojas et al. 2012) encontraron que los sistemas de cultivo bajo sombra presentan un efecto positivo para la transformación del paisaje, la biodiversidad y las especies exóticas invasoras, porque aportan a la conservación de los ecosistemas en cuanto a cobertura vegetal, composición de suelos y, asimismo, a la preservación de las especies nativas de la región cafetera. El cultivo bajo sombra, por ser un sistema de producción más amigable con el entorno, puede llegar a reestablecer suelos erosionados o perdidos, dado que logra adaptar características similares a las de los bosques mesófilos y, además, generar una alternativa para que las comunidades campesinas mejoren su calidad de vida.

Con estas consideraciones, el objetivo de este trabajo fue caracterizar los sistemas productivos de café en los ecotopos $220 \mathrm{~A}$ y $221 \mathrm{~A}$, localizados en el departamento de Nariño, con lo cual, se pretende contribuir al conocimiento y al desarrollo del cultivo del café, en el sur de Colombia.

\section{MATERIALES Y MÉTODOS}

La investigación, se llevó a cabo en los municipios de La Unión y Buesaco, pertenecientes al ecotopo 220A y en los 
municipios de Sandoná, Consacá y La Florida, localizados en el ecotopo 221A, del departamento de Nariño.

Inicialmente, se revisaron las estadísticas de la Federación Nacional de Cafeteros de Nariño, lo que permitió conformar un marco muestral de 16.767 unidades productivas cafeteras (UPC), en los dos ecotopos. Con base en este marco, se estableció un tamaño de muestra de 159 fincas cafeteras. localizadas en los municipios de La Unión y Buesaco (Ecotopo 220) y Sandoná, Consacá y La Florida (Ecotopo 221), mediante la utilización de la fórmula propuesta por Scheaffer et al. (2007).

Posteriormente, la muestra se estratificó con base en el porcentaje de UPC, correspondiente a cada uno de los municipios citados, obteniéndose la siguiente conformación de las encuestas: La Unión, 57; Buesaco, 29; Sandoná, 27; La Florida, 21 y Consacá, 25. En cada municipio, las encuestas se asignaron aleatoriamente entre productores, cuyos predios estaban localizados según los siguientes rangos altitudinales, menor a $1.500 \mathrm{msnm}$, entre 1.501 y $1.800 \mathrm{msnm}$ y mayor a $1.800 \mathrm{msnm}$.

La aplicación del formulario de encuesta, se realizó mediante entrevista personal; además, se hicieron recorridos en las UPC, con el objeto de tener un conocimiento más detallado y real de los sistemas productivos del cultivo de café en las zonas. Todas las respuestas del cuestionario (120), se consideraron variables y se diseñaron en forma categórica, para facilitar la aplicación del método multivariado de análisis de correspondencias múltiples (ACM) y el análisis de clasificación, con base en las distancias de Ward, utilizando el software SPAD 5.6 (Ríos et al. 2004; Chávez et al. 2010).

\section{RESULTADOS Y DISCUSIÓN}

Ecotopo 220A. El ACM del ecotopo 220A mostró una clasificación de la composición porcentual de los cultivos de café, acorde con la altitud de las explotaciones. El 70,9\%, se encuentran localizados entre los 1.500 y los $2.000 \mathrm{msnm}$, mientras que el $12,8 \%$ están por debajo de los $1.500 \mathrm{msnm}$ y el $16,3 \%$ por encima de los $2.000 \mathrm{msnm}$. Solamente, el $31,4 \%$ de los predios realizan análisis de suelos y es una característica similar en los municipios de Buesaco y La Unión.

Los cultivos menores a una hectárea, se encontraron en un $20,7 \%$ de los predios en Buesaco y en un 19,3\%, en el municipio de La Unión. El 10,3\% de los cultivos de Buesaco poseen más de tres hectáreas, mientras que en La Unión, solo el 3,5\% son áreas grandes (mayor a 3ha). El área de los cultivos de café en la zona es de minifundio, está en concordancia con el área de las unidades productivas de café en los principales departamentos cafeteros de Colombia, donde oscilan entre 0,1 y 6,9ha, con un promedio de 2,1 ha (Dussán et al. 2006).

Por otro lado, las fincas con alto volumen de producción de café, correspondieron al 22,2\% de las UPC, en Buesaco y al $77,8 \%$, en La Unión (77,8\%). De las UPC existentes en La Unión, el 71,4\% no reportaron producción, debido a que poseían cultivos nuevos. Es de aclarar, que las UPC se clasificaron en las siguientes categorías: menos de 50 arrobas de producción anual, como bajo volumen de producción; entre 50 y 100 arrobas, correspondían a volumen medio de producción; más de 100 arrobas, a alto volumen de producción y cultivos nuevos, sin producción.

Según el ACM, el 22,38\% de la variabilidad total se puede explicar, a través de tres factores (Tabla 1). El primer factor está conformado, principalmente, por las variables correspondientes al municipio, altitud y tipo de manejo de plagas y enfermedades; el segundo, por el tamaño de la finca, la productividad del suelo, la edad y tipo de plantación, método de control de malezas y producción cafetera de la finca y el tercer factor, lo conforman, en su mayor proporción, las variables altitud, incorporación y manejo agronómico de frutales, como sombrío.

En la figura 1, se observa una gran concentración de los productores en el origen de los ejes del plano factorial, indicando poca variabilidad, especialmente, en las fincas ubicadas en el municipio de La Unión, que se destacan por su

Tabla 1. Varianza explicada por cada valor propio del ACM de las variables categorizadas, correspondientes a encuestas en los ecotopos 220A y 221A.

\begin{tabular}{|c|c|c|c|c|c|c|}
\hline Factor & \multicolumn{2}{|c|}{ Ecotopo 220A } & \multicolumn{3}{c|}{ Ecotopo 221A } \\
\hline No. & VP & V(\%) & VA(\%) & VP & V(\%) & VA(\%) \\
\hline 1 & 0,17 & 8,99 & 8,99 & 0,61 & 8,37 & 8,37 \\
\hline 2 & 0,13 & 6,96 & 15,95 & 0,14 & 7,23 & 15,60 \\
\hline 3 & 0,11 & 6,03 & 22,00 & 0,13 & 6,99 & 22,58 \\
\hline
\end{tabular}

$\mathrm{VP}=$ valor propio, $\mathrm{V}=$ varianza, $\mathrm{VA}=$ varianza acumulada- 
localización por debajo de los 1.800msnm, alta proporción de cultivos nuevos, que no entran en producción y estar asociados con frutales. Más alejado del origen de los ejes, se ubica el municipio de Buesaco, cuyas unidades productivas se sitúan en altitudes superiores a los $1.800 \mathrm{msnm}$.
La proximidad gráfica observada entre individuos y variables, como altitud y producción, explica la naturaleza de la variabilidad dentro y entre grupos; de ahí, que si una de esas características aparece asociada con una finca cafetera, lo más probable es que las características correlacionadas con ella

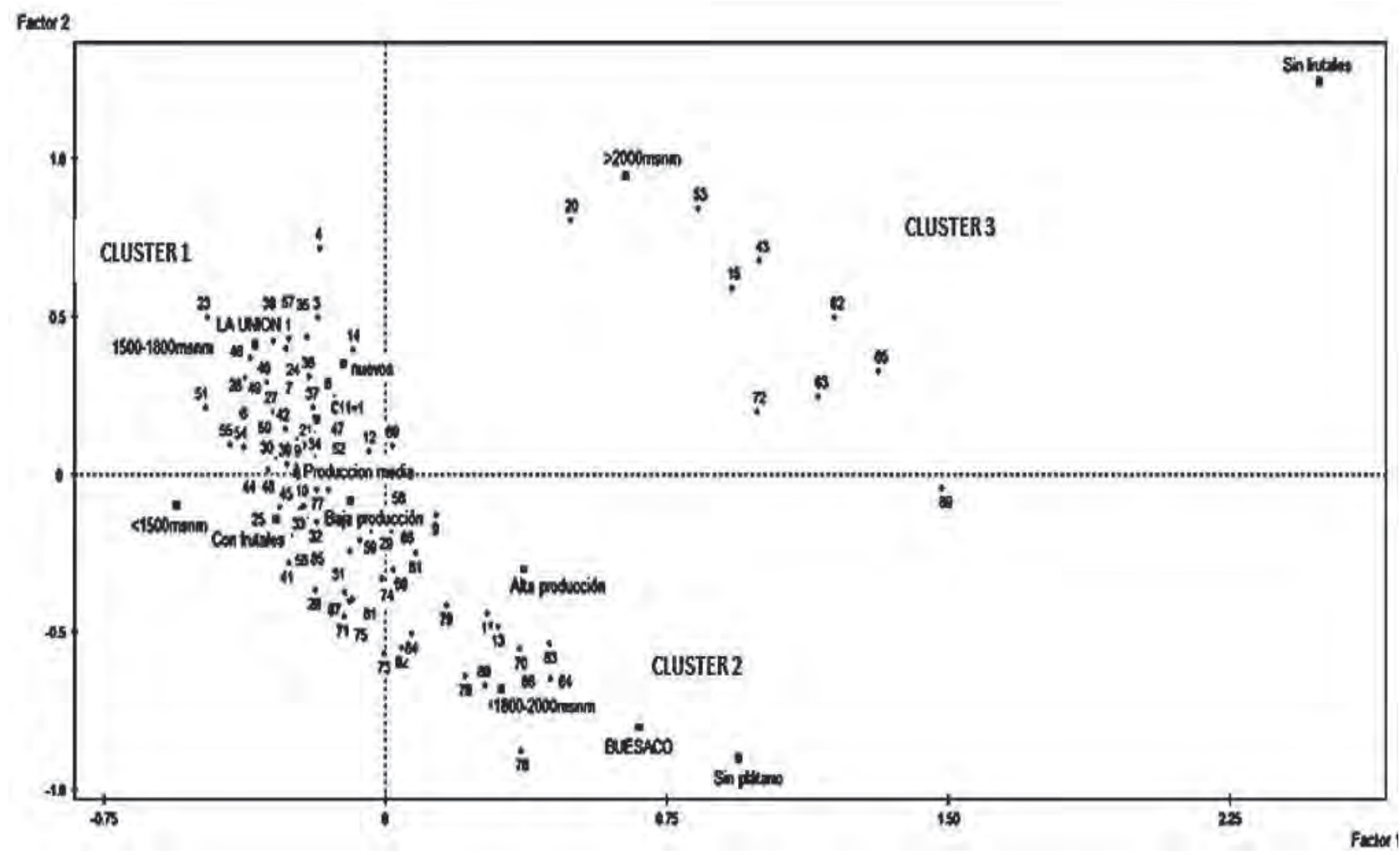

Figura 1. Ubicación de las explotaciones cafeteras del ecotopo 220A y de algunas variables importantes, según su aporte en la conformación de los dos primeros factores del ACM.

también estén presentes en el cultivo (Smith et al. 2002). Las modalidades o individuos (productores) localizadas cerca al origen del plano, no aportan significativamente a la variabilidad del sistema, representan a la población promedio y no permiten discriminarlos entre los grupos formados por el ACM (Carrillo et al. 2011).

El análisis de clasificación permitió identificar tres grandes grupos, que se indican en la tabla 2 . El grupo uno está conformado por 56 productores, que representan el $65,11 \%$ de las UPC que, en su mayoría, pertenecen al municipio de La Unión, que participa con el $58,14 \%$ del grupo; estas unidades productivas, se localizan entre 1.500 y $1.800 \mathrm{msnm}$. Se caracterizan por cultivar plátano y frutales intercalados con el café y por el uso de herbicidas para el manejo de arvenses.
El grupo dos está formado por 21 productores, que corresponde al 24,42\%; Buesaco participa en su gran mayoría en la conformación de este grupo, con el $20,94 \%$; los predios cafeteros, se encuentran localizados entre los 1.800 y los $2.000 \mathrm{msnm}$ y realizan aplicaciones de insecticidas, herbicidas y usan fertilizantes químicos. En general, los cultivos de este grupo son nuevos y los productores hacen uso del crédito bancario, para llevar a cabo sus cultivos. El grupo tres incluye nueve UPC $(10,47 \%)$, de las cuales, cuatro pertenecen a La Unión y cinco a Buesaco, localizadas por encima de los $2.000 \mathrm{msnm}$ y no incluyen frutales en el cultivo de café.

En la figura 1, se muestra la ubicación de los productores según su aporte en la conformación de los dos primeros factores. La mayoría de las fincas, se ubican en el grupo uno y esto significa, una mayor homogeneidad, por su ubicación 
central, mientras que los grupos dos y tres se colocan en un extremo, muy alejadas del grupo uno, con fincas cuyos cultivos de café poseen características que se alejan del promedio, pero sin mucha diferencia entre estos dos grupos.

En el grupo uno predominan los sombríos con árboles de guamo (Inga sp.), en el municipio de La Unión, mientras que en Buesaco, los sombríos predominantes son los cítricos, frutales que hacen un aporte significativo adicional a la economía del productor. La utilización cítricos y plátano, como sombrío en el cultivo de café, garantizan la seguridad alimentaria familiar y generan ingresos alternos, que evitan la dependencia del cultivo del café (Moreno, 2013) y disminuyen el riesgo cuando los precios de grano son bajos.

Tabla 2. Grupos conformados por unidades productivas de café del ecotopo 220A, en el departamento de Nariño.

\begin{tabular}{|c|l|l|c|c|}
\hline Grupo & \multicolumn{1}{|c|}{ No. de predio } & \multicolumn{1}{|c|}{ Ubicación } & $\begin{array}{c}\text { Municipio dentro } \\
\text { del grupo (\%) }\end{array}$ & $\begin{array}{c}\text { Grupo } \\
\mathbf{( \% )}\end{array}$ \\
\hline 1 & $\begin{array}{l}1-8,10,12,14,16,17-19,21-42, \\
44-52,54-57\end{array}$ & LA UNIÓN & 58,14 & 65,11 \\
\hline & $60,66,67,71,77,85$. & BUESACO & 6,97 & \\
\hline 2 & $9,11,13$ & LA UNIÓN & 3,48 & 24,22 \\
\hline & $\begin{array}{l}58,59,61,64,68,70,73-76,78-84, \\
86\end{array}$ & BUESCACO & 20,94 & \\
\hline 3 & $15,20,43,53$ & LA UNIÓN & 4,65 & 10,47 \\
\hline & $62,63,65,69,72$ & BUESACO & 5,82 & \\
\hline
\end{tabular}

Ecotopo 221A. El 58,9\% de los cultivos de café del ecotopo $221 \mathrm{~A}$ están por debajo de los $1.800 \mathrm{msnm}$, mientras que el $21,9 \%$ están entre los 1.800 y los 2.000 msnm y el $19,2 \%$, por encima de los $2.000 \mathrm{msnm}$. Esto coincide con Gamboa et al. (2015), quienes afirman que en la zona sur de Colombia, se produce café de altura y a menor temperatura, lo cual, se traduce en una mayor acidez y calidad de taza, con características especiales que los hacen de gran aprecio en los mercados internacionales; sin embargo, de acuerdo con Oberthür et al. (2011) existen diferencias regionales en la calidad del café, producido entre los departamentos del Cauca y Nariño, definidas por las características ambientales, con excepción de la altitud. Esto indica que las variables relacionadas con los microclimas, la pendiente de las UPC y el suelo, pueden ser determinantes para obtener determinada calidad de café.

La altura sobre el nivel del mar puede afectar la producción y la calidad de café. Se conoce que el tamaño del grano puede crecer con el aumento de la altura, lo contrario ocurre en zonas bajas. Con respecto a la calidad de tasa, no existe una correlación entre la altura definida, pero cafetales de altura con sombrío están asociados a baja calidad de tasa y cafetales bajos con sombrío, se correlacionan con una buena tasa (Bosselmann et al. 2009). En zonas de altura, las poblaciones de la broca del café son bajas, contrariamente con lo que ocurre en la zona bajas, donde la Hypothenemus hampei es una plaga limitante para la producción (Bosselmann et al. 2009).
En el ecotopo 221A predominan los cultivos de 1 a 3 ha $(47,9 \%)$, un $35,6 \%$ son minifundios con menos de una hectárea y solo el $16,4 \%$ son cultivos que superan las tres hectáreas. Generalmente, en el 91,8\% de los cafetales se encuentran árboles frutales y plátano; solo un $9,2 \%$ son monocultivos. El $42,5 \%$ fertiliza con base en el análisis de suelos, mientras que el $57,5 \%$ aplica los fertilizantes, sin una base diagnóstica del suelo.

La experiencia de los cultivadores de café es una gran fortaleza en la región, dado que el 92,9\% de los productores tiene una tradición cafetera por más de diez años y solo el $11 \%$ tiene una experiencia menor a cinco años. En cuanto al volumen productivo de las fincas, el $49,3 \%$ tienen una baja producción (menos de 50 arrobas); el 24,7\%, media (entre 50 y 100 arrobas) y sólo un $11,0 \%$ presentan una producción alta (más de 100 arrobas). El 15,1\% restante corresponde a cultivos nuevos, que todavía no registran producción.

Para la obtención de óptimas producciones y buenas tasas es necesario realizar un buen plan de fertilización, ya sea de origen inorgánico u orgánico, para compensar la extracción que ejerce el cultivo, principalmente elementos, tales como el nitrógeno, el fósforo, el potasio, el calcio y el magnesio (Ramírez et al. 2002; Amaral et al. 2010; Haggar et al. 2011); no obstante, es posible que por los altos costos que representa el uso de los fertilizantes en el cultivo del café, 
los agricultores se vean limitados en la utilización de este insumo. En Colombia, los costos de los fertilizantes en el cultivo representan entre el 10 y el $15 \%$ de los costos totales de la producción y entre el 80 y $90 \%$ de los insumos utilizados (Sadeghian, 2013). Aunque es posible disminuir el uso de fertilizantes en el cultivo del café, con dosis moderadas de fertilización, obteniendo viabilidad económica (Campos et al. 2006).

Según Sadeghian (2013), mediamente los análisis de suelos es posible conocer la fertilidad de los suelos, el plan general de nutrición de los cafetales y la aplicación de enmiendas, lo que disminuye los riesgos económicos y ambientales. La fertilización en los cafetales es fundamental para obtener buenos rendimientos; cuando no se realiza esta labor, la producción se disminuye en más del $40 \%$ de la producción.

El ACM (Tabla 1) mostró que el 22,58\% de la variabilidad total se puede explicar por los tres primeros factores. Entre las variables con mayor asociación con el primer factor, se encuentran el municipio, la altitud y el tipo de fertilizante empleado en la siembra; el segundo factor, lo conforman, principalmente, las variables cultivo de café intercalado con frutales, manejo de los frutales, renovación de cafetales y producción por finca. Las variables que más aportan a la conformación del factor tres son el área del cultivo, número de jornales empleados en el proceso productivo, el conoci- miento de las buenas prácticas agrícolas y la producción por finca.

Existe una alta similitud entre Sandoná y Consacá, mientras que La Florida, se encuentra distante de estos dos municipios en el plano cartesiano, conformado por los dos primeros factores, debido, primariamente, a la mayor altitud de sus cultivos (Figura 2). De igual manera, cuando se ubica en el plano la variable producción/finca, se pudo establecer que las altas producciones están asociadas al municipio de Sandoná, mientras que las bajas y medias, se encuentran más próximas al municipio de Consacá y La Florida, respectivamente.

El análisis de agrupamiento permitió la formación de cuatro grupos definidos por sus principales características (Tabla 3 y Figura 2). El grupo uno, conformado por tres fincas productoras de los municipios de Sandoná y cuatro de Consacá, ubicadas a menos de $1.800 \mathrm{msnm}$, sin asociación de frutales o plátano; representan el 9,58\% de los productores.

El grupo dos, lo componen 32 productores, cuyas UPC se distribuyen en los municipios de Sandoná (24,66\%), Consacá $(17,80 \%)$ y La Florida $(2,74 \%)$; estos productores no reciben la asistencia técnica, lo que trae como consecuencia, que no conozcan la normatividad de las Buenas Prácticas Agrícolas; se localizan en zonas con vientos moderados. El

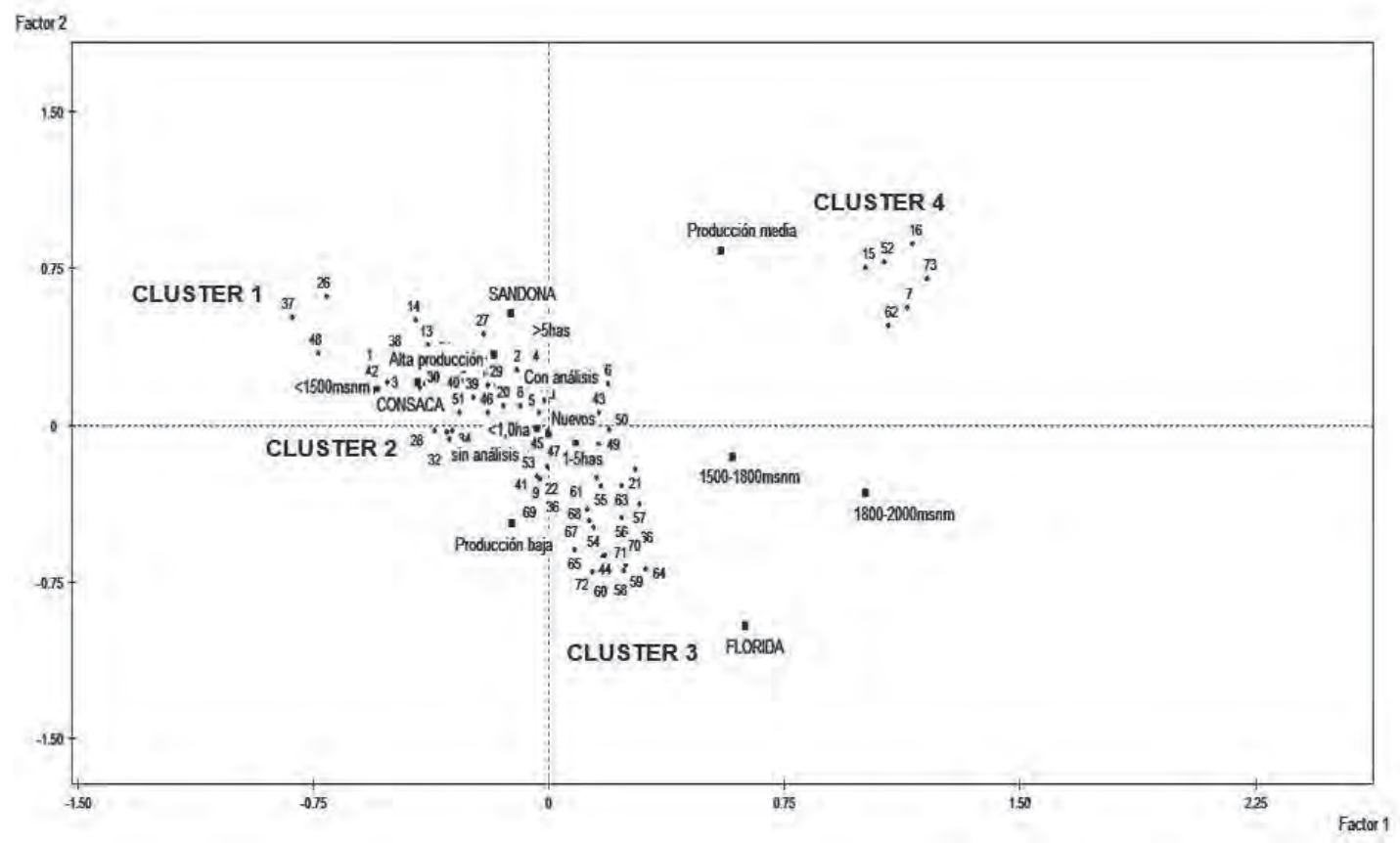

Figura 2. Ubicación de las UPC del ecotopo 221A y de algunas variables importantes, según su aporte en la conformación de los dos primeros factores del ACM. 
Tabla 3. Grupos conformados por unidades productivas de café del ecotopo 221A, en el departamento de Nariño.

\begin{tabular}{|c|l|l|c|c|}
\hline Grupo & \multicolumn{1}{|c|}{ No. de predio } & \multicolumn{1}{|c|}{ Ubicación } & $\begin{array}{c}\text { Municipio dentro } \\
\text { del grupo (\%) }\end{array}$ & $\begin{array}{c}\text { Grupo } \\
\text { (\%) }\end{array}$ \\
\hline 1 & $1,3,26$ & SANDONA & 4,11 & 9,58 \\
\hline & $28,37,42,48$ & CONSACA & 5,47 & \\
\hline 2 & $2,4,5,6,8,10-14,17-20,23,25$, & SANDONA & 24,66 & 45,20 \\
\hline & 27 & CONSACA & 17,80 & \\
\hline & $29-35,38-40,45,46,51$ & LA FLORIDA & 2,74 & \\
\hline 3 & 53,69 & SANDONA & 2,74 & 36,99 \\
\hline & 9,21 & CONSACA & 10,96 & \\
\hline & $53,36,41,43,44,47,49,50$ & LA FLORIDA & 23,29 & \\
\hline 4 & $7,15,16$ & SANDONA & 4,11 & 8,22 \\
\hline & 52 & CONSACA & 1,37 & \\
\hline & 62,73 & LA FLORIDA & 2,74 & \\
\hline
\end{tabular}

grupo tres, con 17 UPC, distribuidas en Sandoná (2,74\%), Consacá (10,96\%) y La Florida (23,29\%), se encuentran en zonas de vientos fuertes y cafetales renovados por soca y, finalmente el grupo cuatro, se caracteriza por monocultivos y ausencia de frutales, como arboles intercalados con el café (Tabla 3).

En los cultivos de café del ecotopo 220A son frecuentes los sombríos de guamo y de cítricos, mientras que en el ecotopo 221A predominan los cultivos con plátano y con anano, así como los cultivos sin sombra. En ambos ecotopos, la mayoría de productores fertilizan sin tener en cuenta el análisis de suelo. La asociación de los sistemas de producción de café con una zona geográfica en particular, permite articular las relaciones entre los diferentes agentes socioeconómicos, la actividad productiva y el ambiente.

Dependiendo de las condiciones agroecológicas es posible implementar cultivos de café a plena exposición. Mediante este sistema, se hacen más eficientes los factores de producción, por lo tanto, es posible obtener altas producciones de café por hectárea y aumentar la rentabilidad del agricultor (Ramírez et al. 2013); sin embargo, la implementación de este sistema de cultivo requiere constante disponibilidad hídrica y de recursos económicos, para suplir las exigencias de insumos que requiere el cultivo. Además, con este tipo de sistema, se disminuye la biodiversidad comparados con cafetales en sistemas agroforestales, donde la producción es menor, pero con grandes ventajas, como la disminución de la evapotranspiración, los requerimientos de agua son menores, existe mayor aporte de materia orgánica, menor riesgo de erosión y mayor sostenibilidad ante la variabilidad climática, entre otras (Farfán, 2013).

La utilización del sombrío altera el microclima del cultivo, modifica el crecimiento y el desarrollo de las plantas, tal como lo plantean Campanha et al. (2004); en algunas condiciones, según Soto et al. (2000), el sombrío puede favorecer la productividad de los cultivos, mientras que en condiciones óptimas para el cultivo, puede causar efectos negativos sobre el rendimiento.

Agradecimientos: Los autores agradecen a la Universidad de Nariño, al departamento de Nariño y al Sistema General de Regalías. Conflicto de intereses: El manuscrito fue reparado y revisado con la participación de todos los autores, quienes declaramos que no existe conflicto de intereses, que ponga en riesgo la validez de los resultados presentados. Financiación: Este estudio fue financiado por el Sistema General de Regalías, el departamento de Nariño y la Universidad de Nariño.

\section{BIBLIOGRAFÍA}

1. AGRONET. 2015. Red de información y comunicación del sectoragropecuario. Disponible desde Internet en: http://www.agronet.gov.co/Paginas/estadisticas. aspx (con acceso 16/04/2016). 
2. AMARAL, J.F.T.D.; MARTINEZ, H.E.P.; LAVIOLA, B.G.; FERNANDES FILHO, E.I.; CRUZ, C.D. 2010. Bean production efficiency and relative allocation of nutrients of four coffee varieties. Rev. Ceres. 57:253262.

3. BOSSELMANN, A.S.; DONS, K.; OBERTHÜR, T.; OLSEN, C.S.; RÆBILD, A.; USMA, H. 2009. The influence of shade trees on coffee quality in small holder coffee agroforestry systems in Southern Colombia. Agric. Ecosyst. Environ. 129(1):253-260.

4. CAMPANHA, M.; SILVA, R.; DE FREITAS, B.; PRIETO, H.; RIBEIRO, S.; FINGER, F. 2004. Growth and yield of coffee plants in agroforestry and monoculture systems in Minas Gerais, Brazil. Agrofor. Systems. 63:75-82.

5. CAMPOS, F.; FURTINI, A.E.; GONTIJO, P.T.; BARROS, E.D.; PEREIRA, P. 2006. Efficiency of NPK fertilization in coffee dense cropping system in Southern Minas Gerais, Brazil. Coffee Sci. 1:135-142.

6. CARRILLO, B.; MOREIRA, V.; GONZÁLEZ, J. 2011. Caracterización y tipificación de sistemas productivos de leche en la zona centro-sur de Chile: un análisis multivariable. IDESIA (Chile). 29(1):71-81.

7. CHÁVEZ, D.; MIRANDA, I.; VARELA, M.; FERNÁNDEZ, L. 2010. Utilización del análisis de clúster con variables mixtas en la selección de genotipos de maíz (Zea mays L.). Rev. Invest. Operacional. 30(3):209216.

8. DUSSÁN, C.; DUQUE, H.; GONZÁLEZ, J. 2006. Caracterización tecnológica de caficultores de economía campesina, de los principales municipios cafeteros de Colombia. Cenicafé. 57(3):167-186.

9. FARFÁN, V.F. 2013. Establecimiento de sistemas agroforestales con café. En: Gast, F.; Benavides, P.; Sanz, J.R.; Herrera, J.C.; Ramírez, V.H; Cristancho, M.A.; Marín, S.M. (eds). Manual del cafetero colombiano Investigación y tecnología para la sostenibilidad en la de la caficultura, Tomo II. Ed. Federación Nacional de Cafeteros de Colombia, Legis, Bogotá D.C. p.4463.

10. FEDERACIÓN NACIONAL DE CAFETEROS DE COLOMBIA -FNC-. 1991. Ecotopos cafeteros de Colombia. Ed. FNC, Bogotá D.C. p.75-76.

11. GAMBOA, R.; MOSQUERA, S.; PAZ, I. 2015. Caracterización física de café especial (Coffea arabica) en el municipio de Chachagüí (Nariño, Colombia. Rev. Lasallista Invest. 12(1):90-98.

12. GÓMEZ G.; CABALLERO, R.; BALDIÓN R. 1991. Ecotopos Cafeteros. Federación Nacional de Cafeteros de Colombia: Cenicafé - Agroclimatología, División de Desarrollo Social. Bogotá. 125p.

13. HAGGAR, J.; BARRIOS, M.; BOLAÑOS, M.; MERLO, M.; MORAGA, P.; MUNGUIA, R.; PONCE, A.; ROMERO, S.; SOTO, G.; STAVER, C.; VIRGINIO, E.D.M.F. 2011. Coffee agroecosystem performance under full sun, shade, conventional and organic management regimens Central America. Agroforest. Syst. 82:285301.

14. MERMA, I.; JULCA, A. 2012. Caracterización y evaluación de la sustentabilidad de fincas en alto Urubamba, Cusco, Perú. Ecología Aplicada. 11(1):1-11.

15. MORENO, B.M.A. 2013. Sistemas de producción de café en arreglos interespecíficos. En: Gast, F.; Benavides, P.; Sanz, J.R.; Herrera, J.C.; Ramírez, V.H; Cristancho, M.A.; Marín, S.M. (Eds). Manual del cafetero colombiano Investigación y tecnología para la sostenibilidad en la de la caficultura, Tomo II. Ed. Federación Nacional de Cafeteros de Colombia, Legis, Bogotá D.C. p.64-84.

16. OBERTHÜR, T.; LÄDERACH, P.; POSADA, H.; FISHER, M.J.; SAMPER, L.F.; ILLERA, J.; JARVIS, A. 2011. Regional relationships between inherent coffee quality and growing environment for denomination of origin labels in Nariño and Cauca, Colombia. Food policy. 36(6):783-794.

17. ORGANIZACIÓN INTERNACIONAL DEL CAFÉ -OIC-. 2014. Comercio mundial del café (1963-2013): reseña de los mercados, retos y oportunidades con que se enfrenta el sector. Consejo Internacional del café, Londres, UK. 27p.

18. RAMÍREZ, F.; BERTSCH, F.; MORA, L. 2002. Consumo de nutrimentos por los frutos y bandolas de café caturra durante un ciclo de desarrollo y maduración en Aquiares, Turrialba, Costa Rica. Agron. Costarricense 26(1):33-42.

19. RAMÍREZ, H.; JARAMILLO, A.; PEÑA, J. 2013. Gestión del riesgo agroclimático: vulnerabilidad y capacidad de adaptación del sistema de producción de café. En: Gast, F.; Benavides, P.; Sanz, J.R.; Herrera, J.C.; Ramírez, V.H; Cristancho, M.A.; MARÍN, S.M. (Eds). Manual del cafetero colombiano Investigación y tec- 
nología para la sostenibilidad en la de la caficultura, Tomo I. Ed. Federación Nacional de Cafeteros de Colombia, Legis, Bogotá D.C. p.91-114.

20. RÍOS, G.; ROMERO, M.; BOTERO, M.; FRANCO, G.; PÉREZ, J.; MORALES, J.; GALLEGO, J.; ECHEVERRY, D. 2004. Zonificación, caracterización y tipificación de los sistemas de producción de lulo (Solanum quitoense Lam) en el Eje Cafetero. Rev. CORPOICA. 5(1):22-30.

21. ROJAS, A.; HARTMAN, K.; ALMONACID, R. 2012. El impacto de la producción de café sobre la biodiversidad, la transformación del paisaje y las especies exóticas invasoras. Ambiente y Desarrollo. 16(30):93104.

22. SADEGHIAN, K.S. 2013. Nutrición de los cafetales. En: Gast, F.; Benavides, P.; Sanz, J.R.; Herrera, J.C.; Ramírez, V.H; Cristancho, M.A.; Marín, S.M. (eds). Manual del cafetero colombiano Investigación y tecnología para la sostenibilidad en la de la caficultura, Tomo II. Ed. Federación Nacional de Cafeteros de Colombia, Legis, Bogotá D.C. p.85-116.

23. SALDÍAS, C. 2013. El servicio de extensión acompañado a la investigación para una mejor atención a los cafeteros de Colombia, En: Gast, F.; Benavides, P.; Sanz, J.R.; Herrera, J.C.; Ramírez, V.H; Cristancho, M.A.; Marín, S.M. (eds). Manual del cafetero colombiano Investigación y tecnología para la sostenibilidad en la de la caficultura, Tomo I. Ed. Federación Nacional de Cafeteros de Colombia, Legis, Bogotá D.C. p.47-62.
24. SCHEAFFER, R.; MENDENHALL, W.; LYMAN, O. 2007. Elementos de muestreo. $6^{\text {a }}$ edición. Ed. International Thomson, Madrid. 455p.

25. SMITH, R.; MOREIRA, V.; LATRILLE, L. 2002. Caracterización de sistemas productivos lecheros en la $\mathrm{X}$ región de Chile mediante análisis multivariable. Agricultura Técnica (Chile). 62(3):375-395.

26. SOTO, P.; PERFECTO, I.; CASTILLO, H.; CABALLERO, N. 2000. Shade effect on coffee production at the northern Tzeltal zone of the state of Chiapas, México. Agr. Ecosyst. Environm. 80:61-69.

27. TORRENTE, A.; LADINO, A. 2009. Caracterización de propiedades fisicoquímicas de los suelos de la zona cafetera del municipio de Isnos con el fin de establecer su aptitud de uso y manejo. Rev. Ing. Región. 6(1):77-82.

28. URUEÑA, M. 2013. El mercado mundial y nacional del café en el siglo XXI, En: Gast, F.; Benavides, P.; Sanz, J.R.; Herrera, J.C.; Ramírez, V.H; Cristancho, M.A.; Marín, S.M. (eds). Manual del cafetero colombiano Investigación y tecnología para la sostenibilidad en la de la caficultura, Tomo I. Ed. Federación Nacional de Cafeteros de Colombia, Legis, Bogotá D.C. p.17-25.

Recibido: Enero 18 de 2016

Aceptado: Abril 26 de 2016

Cómo citar:

Criollo Escobar, H.; Lagos Burbano, T.C.; Bacca Ibarra, T.; Muñoz Belalcazar, J.A. 2016. Caracterización de los sistemas productivos de café en Nariño, Colombia. Rev. U.D.C.A Act. \& Div. Cient. 19(1): 105-113. 